\title{
Transrectal Ultrasound Appearance of Granulomatous Prostatitis
}

\author{
Ronald Bude, MD*, Robert L. Bree, MD*t, Ronald S. Adler, MD, PhD*, S. Zafar Jafri, MDł
}

Granulomatous prostatitis is an uncommon condition that can masquerade as prostatic carcinoma on both digital rectal exam and prostate ultrasound. It occurs most often after acute urinary tract infection, transurethral prostate resection, or needle biopsy. It can be seen in systemic granulomatous diseases and after intravesical bacillus Calmette-Guerin (BCG) therapy for bladder carcinoma. In some cases it is idiopathic. Six patients who underwent transrectal ultrasound of the prostate and subsequent transrectal ultrasound-guided biopsy had histologic diagnosis of granulomatous prostatitis. One patient was undergoing BCG therapy for bladder cancer. Two patients had recent urinary tract infections.
The other three patients had no known predisposing conditions. Sonographically, the glands were enlarged in five patients, with multiple large and small hypoechoic zones throughout the peripheral, transition, and central zones. The appearance was similar to that seen in diffuse prostatic carcinoma. In one patient, a solitary hypoechoic lesion in the peripheral zone, indistinguishable from carcinoma, was present. Granulomatous prostatitis should be considered in the differential diagnosis of focal and diffuse abnormality with prostatic ultrasound. KEY WORDS: prostate ultrasound, prostate cancer, prostatitis, prostate inflammation. (f Ultrasound Med 9:677, 1990)
$\mathrm{A}$ lthough the sonographic appearance of the prostate, in general, and in prostatic carcinoma, in particular, has been well described in the recent literature, ${ }^{1-9}$ there has been no description of the appearance of granulomatous prostatitis. Granulomatous prostatitis is an uncommon entity that mimics prostatic carcinoma on rectal examination..$^{10}$ It can occur after urinary tract infection, transurethral prostatectomy, needle biopsy, or intravesical bacillus CalmetteGuerin (BCG) therapy for bladder carcinoma. It occurs in systemic granulomatous diseases and also can be idiopathic. ${ }^{10}$ Most common symptoms are fever, chills, urgency, frequency, and dysuria. ${ }^{10}$ Although symptoms usually subside in a few months, gland induration, when present, results in persistent diffuse abnormality in $80 \%$

Received November 30, 1989, from "University of Michigan Medical Center, Ann Amor, Michigan; Veterans Administration Medical Center, Ann Arbor, Michigan; and \$William Beaumont Hospital, Royal Oak, Michigan. Revised manuscript accepted April 25, 1990.

Address correspondence and reprint requests to Dr. Bree: University of Michigan Medical Center, Department of Radiology, 1500 East Medical Center Drive, Ann Arbor, MI 48109. or a single focal nodule in $20 \% .^{10}$ We report the sonographic appearance of biopsy-proven granulomatous prostatitis, seen in six cases.

\section{METHODS AND MATERIALS}

Among a large series of patients examined with transrectal ultrasound of the prostate, six were discovered to have granulomatous prostatitis confirmed histologically with ultrasound-guided transrectal biopsy. The ultrasound examinations were performed with highfrequency transrectal probes imaging in axial and sagittal planes. When focal or diffuse abnormalities were discovered, ultrasound-guided biopsies were performed using standard biopsy techniques, well described in the literature. ${ }^{1,7}$ The biopsies were all performed using an automatic biopsy device, the Biopty Biopsy Instrument (Bard Urologic, Covington, GA), utilizing prebiopsy and postbiopsy antibiotic coverage.?

The lesions that were visualized and biopsied consisted of discrete hypoechoic foci, indistinguishable so- 
nographically from prostate carcinoma. In patients where there were multiple abnormal areas detected sonographically, at least two separate discrete foci were biopsied. In addition, when a single focal abnormality was detected, a sonographically guided biopsy into a normal area in the contralateral lobe was also performed.

\section{RESULTS}

Six patients with suspected prostatic carcinoma, based on digital rectal exam and findings on prostate sonography, were retrospectively found to have granulomatous prostatitis. The patients ranged in age from 42 to 80 years. One of the patients, a 42 -year-old man, had previous intravesical BCG therapy for bladder carcinoma. Two patients had recent urinary tract infections. Three patients had no known predisposing conditions. Five of the six patients had enlarged lobular glands with multiple hypoechoic lesions involving multiple areas of the prostate gland (Figs. 1 and 2). In one patient, a solitary hypoechoic lesion was present in the peripheral zone (Fig. 3). Biopsies of the hypoechoic zones revealed granulomatous prostatitis in all patients.

Cultures (four patients) or stains (one patient) for acidfast bacilli were obtained in five of the six patients and were negative. No specific treatment for the granulomatous prostatitis was rendered. Four patients were asymptomatic, prior to and following the ultrasound and biopsy. Two patients had obstructive voiding symptoms, clinically attributed to prostatic hypertrophy. No complications from the biopsies were reported.

\section{DISCUSSION}

The clinical and pathologic findings of granulomatous prostatitis are well described by Stillwell et al. ${ }^{10}$ Pathologically, granulomatous prostatitis is a granulomatous inflammatory reaction, usually noncaseating. The pathogenesis begins with prostatic duct blockage from infection or surgical trauma, causing tissue necrosis. $\mathrm{Ne}$ crosis allows cellular debris and prostatic secretions, including corpora amylacea, to escape into the interstitial tissue, inciting an intense granulomatous reaction. Diffuse involvement is seen in $80 \%$ of cases, but in $20 \%$, discrete foci can be found. The most common symptoms are fever, chills, and irritable bladder signs, such as dysuria, frequency, and urgency. Patients are usually $\mathbf{5 0}$ to 70 years in age. In our series, four patients were asymptomatic at the time of ultrasound and biopsy, and two had obstructive voiding symptoms (hesitancy, nocturia, and urgency).

In approximately $70 \%$ of cases, there is no defined etiology for granulomatous prostatitis. The most common predisposing factor is a recent urinary tract infection such as acute cystitis or acute prostatitis. In about $\mathbf{2 5 \%}$ of cases, a prior biopsy or transurethral resection of the prostate will have been performed. A direct connection between these procedures and the development of granulomatous prostatitis has not been definitely proven. ${ }^{10}$ Granulomatous prostatitis can be seen in systemic granulomatous diseases such as Wegener's granulomatosis or allergic granulomatosis. Specific granulomatous infections such as tuberculosis, syphilis, and intravesical BCG therapy can lead to granulomatous prostatitis. ${ }^{\text {In }}$ our series, one patient had been treated

Figure 1 Transverse scan through the midportion of the gland in a 43 year-old man who was previously treated for transitional cell carcinoma with intravesical bacillus Calmette-Guerin therapy. The gland is moderately enlarged and there are multiple focal hypoechoic lesions seen throughout. There are scattered bright echoes compatible with corpora amylacea and/or calci* fications. Biopsy of two of the hypoechoic lesions revealed granulomatous prostatitis.

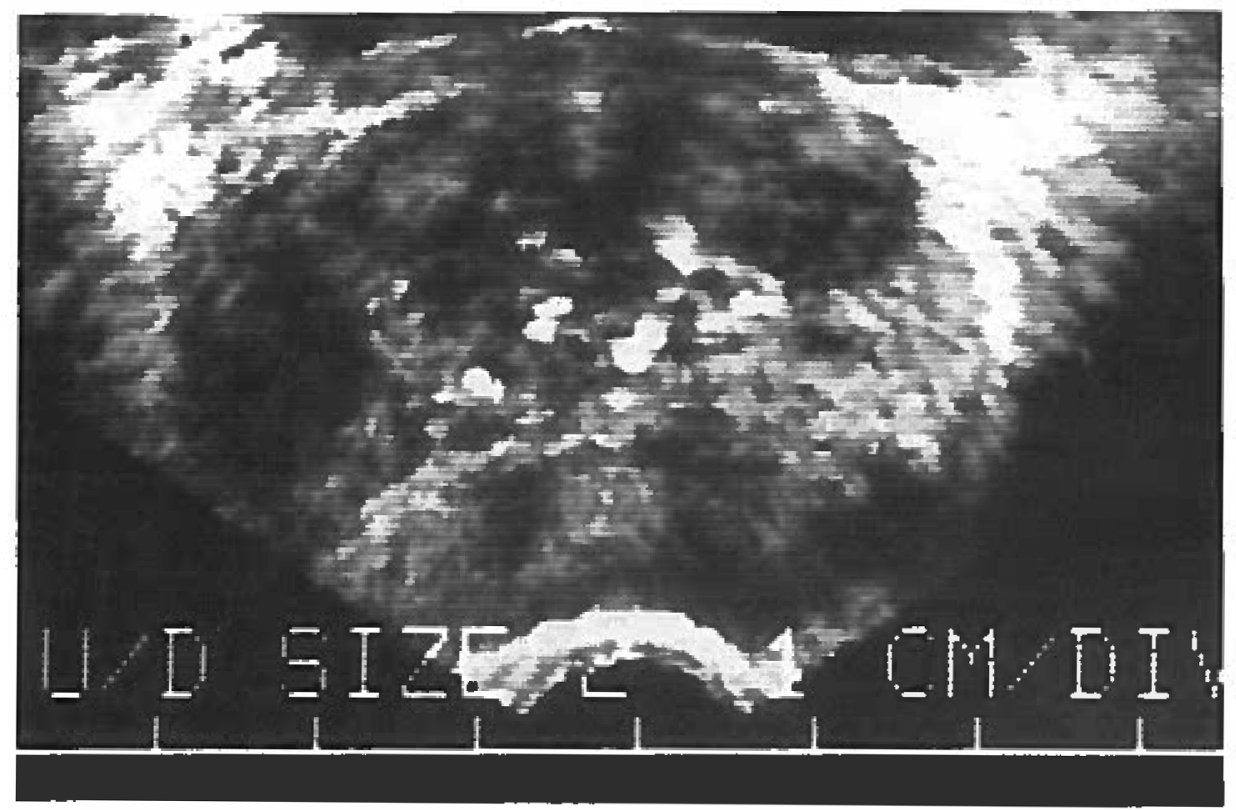




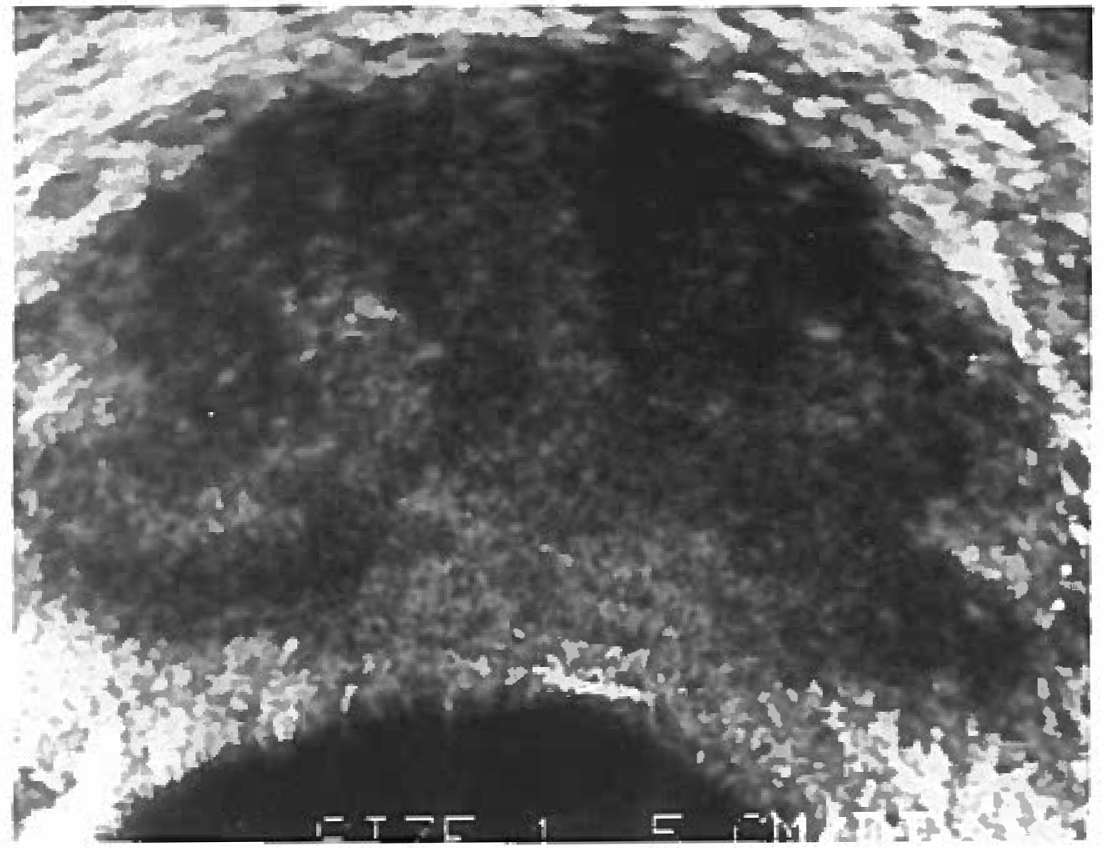

Figure 2 An 80-year-old man was examined because of a palpably abnormal prostate with diffuse induration. Transverse scan demonstrates prostatic enlargement with hypoechoic nodules throughout. Biopsy performed in both lobes revealed granulomatous prostatitis. with BCG therapy for bladder carcinoma. Two patients had recent urinary tract infections 4 and 6 weeks prior to the discovery of granulomatous prostatitis.

Clinically, approximately $60 \%$ of patients with granulomatous prostatitis have a rectal examination that mimics prostatic carcinoma. This may be a discrete nodule or a diffusely indurated gland. In the remainder of patients, palpable findings are felt to be benign. The palpable findings are slow to resolve and may remain for years. Serum acid phosphatase elevation may oc- cur if gland infarction is present. ${ }^{12}$ In our series, two patients presented with a small palpable prostatic nodule, one presented with a firm right lobe, and the remainder presented with diffusely abnormal indurated glands.

Sonographically, five of the six patients presented with enlarged glands with multiple discrete hypoechoic lesions in all three prostatic zones (peripheral, transition, and central; Figs. 1 and 2). One patient presented with a solitary hypoechoic peripheral zone lesion (Fig.

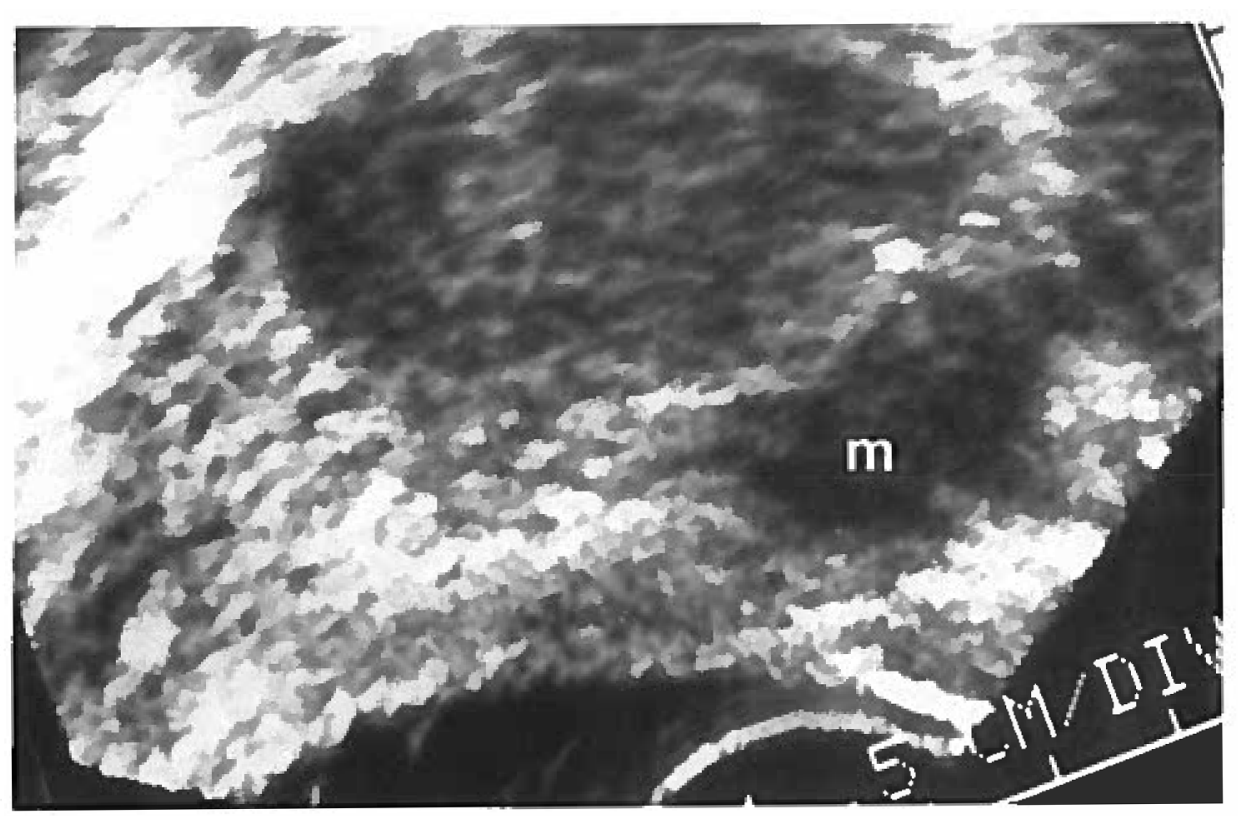

Figure 3 Sagittal transrectal sonogram of the right lobe of the prostate in a 70-year-old patient with a palpable nodule. A hypoechoic mass (m) is identified near the prostatic apex. Transrectal ultrasound-guided biopsy of this mass revealed granulomatous prostatitis. In addition, a biopsy of the opposite lobe that was not felt to be sonographically abnormal, also revealed granulomatous prostatitis. 
Figure 4 Photomicrograph of representative biopsy samples showing homogeneous nature of the granulomatous disease within the prostate and explaining the hypoechoic nature of the lesions on ultrasound.

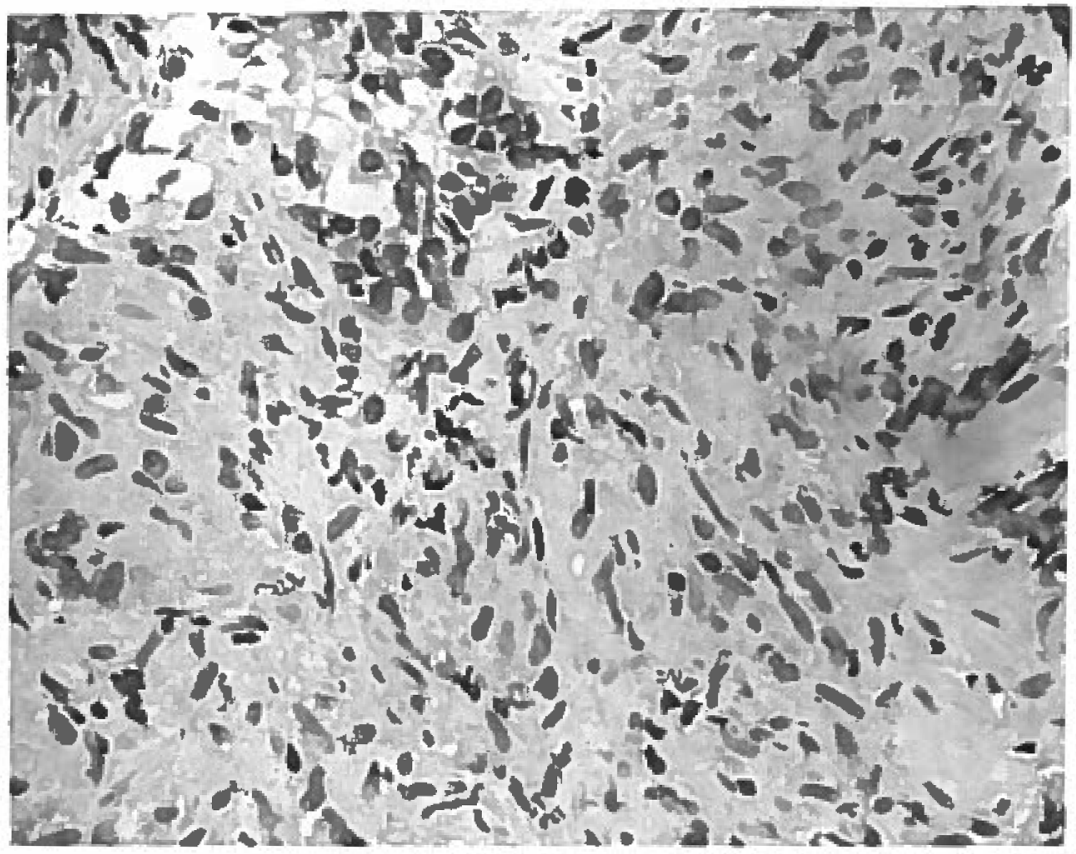

3). Both as a solitary hypoechoic nodule or as diffuse gland involvement, the sonographic appearance was indistinguishable from prostatic carcinoma. This is not surprising, given the nature of granulomatous inflammation to be relatively discrete and homogeneous histologically. ${ }^{10,12}$ The homogeneous nature of the granulomatous inflammatory response is demonstrated in one of the biopsy specimens (Fig. 4). Accurate differentiation can be made with ultrasound-guided biopsy, which should be the procedure of choice in these patients.

\section{REFERENCES}

1. Lee F, Littrup PJ, Kumasaka GH, et al: The use of transrectal ultrasound in the diagnosis, guided biopsy, staging and screening of prostate cancer. Radiographics 7:627, 1987

2. Lee F, Gray JM, McLeary RD, et al: Prostatic evaluation by transrectal sonography: Criteria for diagnosis of early carcinoma. Radiology 158:91, 1986

3. Sanders RC, Hamper UM, Dahnert WF: Update on prostatic ultrasound. Urol Radiol 9:110, 1987

4. Rifkin MD, Choi H: Implications of small, peripheral hypoechoic lesions in endorectal US of the prostate. Radiology 166:619, 1988
5. Rifkin MD, McGlynn ET, Choi H: Echogenicity of prostate cancer correlated with histological grade and stromal fibrosis: Endorectal US studies. Radiology 170:549, 1989

6. Hendrikx AJM, Doesburg WH, Reintjes SP, et al: Effectiveness of ultrasound in the preoperative evaluation of patients with prostatism. Prostate 13:199, 1988

7. Torp-Pedersen S, Lee F, Littrup PJ, et al: Transrectal biopsy of the prostate guided with transrectal US: Longitudinal and multiplanar scanning. Radiology 170:23, 1989

8. Lee F, Torp-Pedersen S, Littrup PJ, et al: Hypoechoic lesions of the prostate: Clinical relevance of tumor size, digital rectal examination and prostate specific antigen. Radiology 170:29, 1989

9. Hardt NS, Kaude JV, Li KC, et al: Sonography of the prostate: In vitro correlation of sonographic and anatomic findings in normal glands. AJR 151:955, 1988

10. Stillwell T], Engen DE, Farrow GM: The clinical spectrum of granulomatous prostatitis: A report of 200. J Urol 138+320, 1987

11. Lamm DL, Stogdill VD, Stogdill BJ, et al: Complications of bacillus Calmette-Guerin immunotherapy in 1,278 patients with bladder cancer. J Urol 135:272, 1986

12. Schmidt JD: Non-specific granulomatous prostatitis: Classification, review and report of cases. J Urol 94:607, 1965 